\title{
MATHEMATICAL MODEL OF SOLIDIFICATION OF THE AXISYMMETRIC CASTING WHILE TAKING INTO ACCOUNT ITS SHRINKAGE
}

\author{
Leszek Sowa \\ Institute of Mechanics and Machine Design, Czestochowa University of Technology \\ Czestochowa, Poland \\ sowa@imipkm.pcz.pl
}

\begin{abstract}
In this paper, the mathematical and numerical model of the solidification of a cylindrical shaped casting, while taking into account the process of the mould cavity filling with molten metal, has been proposed. The feeding of the casting by a riser head during solidification and the formation of shrinkage cavity has also been taken into consideration. The shape and depth of the shrinkage cavity distribution was estimated. One aim of the paper is to obtain a casting without shrinkage defects. Velocity fields were obtained by solving the Navier-Stokes equations and the continuity equation, while the thermal fields were obtained by solving the conduction equation containing the convection term. The changes in the thermophysical parameters, with respect to temperature, were taken into account. The problem was solved by the finite element method.
\end{abstract}

Keywords: mathematical modelling, solidification, shrinkage cavity, molten metal flow

\section{Introduction}

Constantly increasing customer demands for the production of high-quality steels result in the intensive technological development of their production. Consistently high quality is not easy to achieve, hence the need for continuous improvement of casting methods. Production of the castings is aimed at providing a highquality product, free from defects. One of the main causes of defects in castings is the phenomenon of shrinkage of the casting. Shrinkage is a phenomenon concerning the reduction in size of a casting during its transition from a liquid to a solid state. This phenomenon also causes the formation of shrinkage cavities and porosity in the casting $[1,2]$. The preventive measure is supplementing the shortage of liquid metal with the risers in appropriate shapes. Today, the dominant method of selection of the size and shape of the riser head is the computer simulation [2-5]. With the increasing power of computer hardware and software, mathematical modelling is becoming an important tool to understand all phenomena which take place in the whole casting cycle. The great number of phenomena in the casting process necessitates the need for continuous research. Thus, there is a need for 
the formulation of coupled mathematical models that take into account the many phenomena occurring in the whole casting process of steel [4-9].

This paper concerns the modelling of the solidification process including the phenomena of heat transfer and fluid flow during the initial stage of the steel casting process in metal moulds. During this period, the molten metal motions have an essential influence on solidification kinetics. An analysis of solidification kinetics, by determining the velocity and temperature fields in a system of riser-castingmetal moulds was made. In many papers, only the heat conductivity equation was solved during the simulation of the solidification process. The mould cavity was assumed to be fully filled by molten metal at pouring temperature as an initial condition for computation. In this case, the influence of the metal motion on the solid phase growth kinetics during the solidification process was neglected $[1-3,6]$. After completion of the filling process the main solidified of molten metal takes place and its shrinkage. The phenomenon of casting shrinkage cannot be avoided. However, it is possible to minimize the occurrence of its negative effects on the casting. One approach is to design the mould in such a way that the solidification proceeds in accordance to the assumed direction [1-5]. At the beginning the surface of the casting solidifies while the core remains liquid. In the final stage the riser solidifies and the shrinkage cavity appears there. Later the riser is truncated. Thanks to this, the shrinkage cavity is removed from the casting. Its shape and localization is variable and depends on the shape of the riser-casting system and the cooling parameters. Numerical modelling of the shrinkage cavity formation is not often discussed in the literature. Examples of papers considering that phenomenon are articles [1-5]. Simulations of only temperature distribution in the solidifying casting are able to provide some indication about a possible location of the shrinkage cavities in the casting $[3,5]$. However, the location of the shrinkage cavities is also affected by many other factors which should be considered in simulations $[1,2]$. Generally, the aim of search is to obtain a casting without shrinkage defects.

\section{Mathematical model of the casting solidification taking into account its shrinkage and the liquid phase motions}

The proposed model for the numerical simulation of solidification gives consideration to both the motions of molten metal during the mould cavity filing process and convective motions after pouring. It is based on solving the following system of differential equations, in a cylindrical axisymmetry coordinate system $[4,5,9]$ :

- the Navier-Stokes equations

$$
\begin{aligned}
& \mu\left(\frac{\partial^{2} v_{r}}{\partial r^{2}}+\frac{1}{r} \frac{\partial v_{r}}{\partial r}+\frac{\partial^{2} v_{r}}{\partial z^{2}}-\frac{v_{r}}{r^{2}}\right)-\frac{\partial p}{\partial r}+\rho g_{r} \beta\left(\Theta-\Theta_{\infty}\right)=\rho \frac{d v_{r}}{d t} \\
& \mu\left(\frac{\partial^{2} v_{z}}{\partial r^{2}}+\frac{1}{r} \frac{\partial v_{z}}{\partial r}+\frac{\partial^{2} v_{z}}{\partial z^{2}}\right)-\frac{\partial p}{\partial z}+\rho g_{z} \beta\left(\Theta-\Theta_{\infty}\right)=\rho \frac{d v_{z}}{d t}
\end{aligned}
$$


- the continuity equation

$$
\frac{\partial_{r}}{\partial r}+\frac{v_{r}}{r}+\frac{\partial_{z}}{\partial z}=0
$$

- the heat conduction equation containing the convection term

$$
\frac{\lambda}{r} \frac{\partial \Theta}{\partial r}+\frac{\partial}{\partial r}\left(\lambda \frac{\partial \Theta}{\partial r}\right)+\frac{\partial}{\partial z}\left(\lambda \frac{\partial \Theta}{\partial z}\right)=\rho c_{e f}\left(\frac{\partial \Theta}{\partial t}+v_{r} \frac{\partial \Theta}{\partial r}+v_{z} \frac{\partial \Theta}{\partial z}\right)
$$

where $\Theta[\mathrm{K}]$ is the temperature, $t[\mathrm{~s}]$ is the time, $\rho=\rho(\Theta)\left[\mathrm{kg} / \mathrm{m}^{3}\right]$ is the density, $\lambda[\mathrm{W} /(\mathrm{mK})]$ is the thermal conductivity coefficient, $v_{r}, v_{z}[\mathrm{~m} / \mathrm{s}]$ are the $r$-component and $z$-component of velocity, respectively, $\mu(\Theta)\left[\mathrm{Ns} / \mathrm{m}^{2}\right]$ is the dynamical viscosity coefficient, $c_{e f}=c+L /\left(\Theta_{L}-\Theta_{S}\right)[\mathrm{J} /(\mathrm{kgK})]$ is the effective specific heat of a mushy zone, $L[\mathrm{~J} / \mathrm{kg}]$ is the latent heat of solidification, $c[\mathrm{~J} /(\mathrm{kgK})]$ is the specific heat, $p\left[\mathrm{~N} / \mathrm{m}^{2}\right]$ is the pressure, $g_{r}, g_{z}\left[\mathrm{~m} / \mathrm{s}^{2}\right]$ are the $r$ - and $z$-component of gravitational acceleration, respectively, $\beta[1 / \mathrm{K}]$ is the volume coefficient of thermal expansion, $r, z[\mathrm{~m}]$ are the coordinates of the vector of the considered node's position, $\Theta_{\infty}[\mathrm{K}]$ is the reference temperature $\Theta_{\infty}=\Theta_{i n}, r[\mathrm{~m}]$ is the radius.

In the applied model of solid phase growth, the internal heat sources do not become evident in the equation of heat conductivity because they are in the effective specific heat of the mushy zone $[2,4-6]$. The system of equations (1)-(3) is completed by the appropriate boundary and initial conditions for temperature and velocity fields [1-9]. The problem was solved by the finite element method [1, 2, 4-7].

The initial conditions for velocity fields and temperature fields are given as:

$$
\mathbf{v}\left(r, z, t_{0}\right)=\mathbf{v}_{0}(r, z)=\left.v_{i n}\right|_{\Gamma_{1-1}}, \quad \Theta\left(r, z, t_{0}\right)=\Theta_{0}(r, z)=\left\{\begin{array}{lll}
\Theta_{i n} & \text { on } & \Gamma_{1-1} \\
\Theta_{A} & \text { in } & \Omega_{A} \\
\Theta_{M} & \text { in } & \Omega_{M}
\end{array}\right.
$$

The boundary conditions on the indicated surfaces (Fig. 1), specified in the considered problem were as follows:

- for velocity

$$
\begin{aligned}
& \left.v_{n}\right|_{\Gamma_{1-1}}=v_{i n},\left.\quad v_{t}\right|_{\Gamma_{1-1}}=\left.v_{t}\right|_{\Gamma_{2-2}}=\left.v_{n}\right|_{\Gamma_{2-2}}=0, \\
& \left.v_{n}\right|_{r=0}=0,\left.\quad v_{n}\right|_{\Gamma_{G}}=0,\left.\quad v_{t}\right|_{\Gamma_{G}}=0,\left.\quad \frac{\partial v_{t}}{\partial n}\right|_{r=0}=0, \\
& \left.v_{n}\right|_{\Gamma_{A L}}=v_{z}^{*}, \quad \text { where } v_{z}^{*}=\frac{d_{1}^{2}}{d_{o}^{2}} v_{i n} \quad \text { or } v_{z}^{*}=\frac{d_{1}^{2}}{d_{n}{ }^{2}} v_{i n} \text {, }
\end{aligned}
$$


- for temperature

$$
\begin{array}{cc}
\left.\Theta\right|_{\Gamma_{1-1}}=\Theta_{i n}, & \left.\frac{\partial \Theta}{\partial n}\right|_{r=0}=0, \\
\left.\frac{\partial \Theta}{\partial n}\right|_{\Gamma_{2-2}}=0, & \left.\lambda_{M} \frac{\partial \Theta_{M}}{\partial n}\right|_{\Gamma_{M}}=-\alpha_{M}\left(\left.\Theta_{M}\right|_{\Gamma_{M}}-\Theta_{a}\right), \\
\left.\lambda_{S} \frac{\partial \Theta_{S}}{\partial n}\right|_{\Gamma_{G-}}=\left.\lambda_{G} \frac{\partial \Theta_{G}}{\partial n}\right|_{\Gamma_{G-}}, & \left.\lambda_{G} \frac{\partial \Theta_{G}}{\partial n}\right|_{\Gamma_{G+}}=\left.\lambda_{M} \frac{\partial \Theta_{M}}{\partial n}\right|_{\Gamma_{G+}},
\end{array}
$$

where $\Theta_{a}[\mathrm{~K}]$ is the ambient temperature, $\alpha_{M}\left[\mathrm{~W} /\left(\mathrm{m}^{2} \mathrm{~K}\right)\right]$ is the heat-transfer coefficient between a mould and ambient, $\Theta_{M}[\mathrm{~K}]$ is the mould temperature, $\Theta_{i n}[\mathrm{~K}]$ is the temperature at the inlet gate, $n$ is the outward unit normal surface vector.

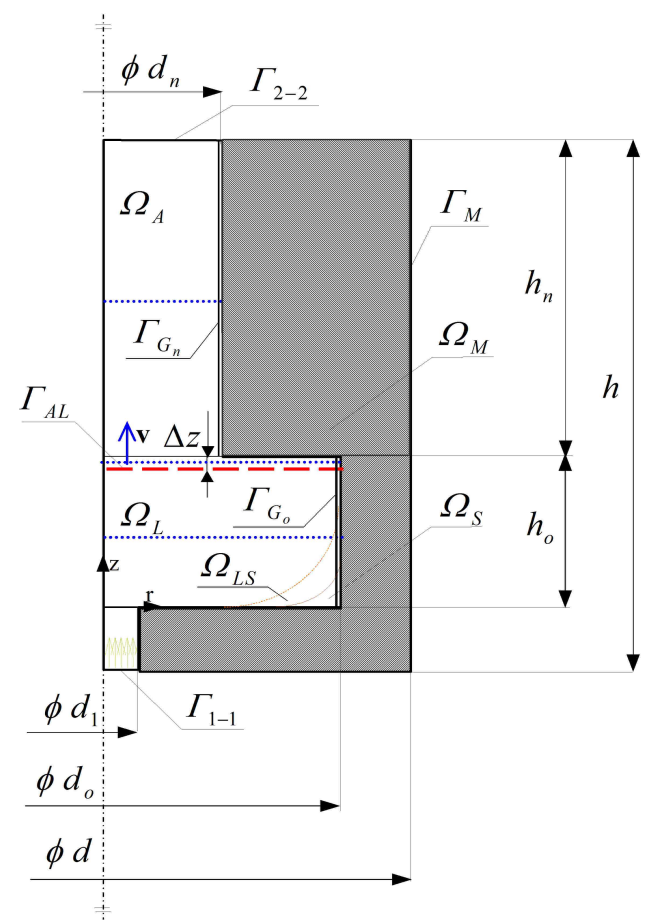

Fig. 1. Scheme and identification of sub-regions of the considered region

Generally, the volume of metal decreases as the temperature falls because the shrinkage phenomenon of metal takes place and the shrinkage cavity is created. The amount of solidification shrinkage is presumed according to the following procedure [2-4]. The analyzed area of the mould cavity is initially filled by molten metal whose volume is equal to the total volume of mould cavity $(V)$. It denotes that:

$$
\sum_{i=1}^{n} V_{L(i)}=V, \quad \sum_{i=1}^{n} V_{S(i)}=0, \quad \sum_{i=1}^{n} V_{s h c(i)}=0,
$$


where $n$ is the total number of nodes, $V_{L(i)}, V_{S(i)}, V_{\text {shc(i) }}\left[\mathrm{m}^{3}\right]$ are the volume of liquid, solid and shrinkage cavity in the $i$-th node, respectively.

During the cooling process, in the liquid state, the nodal temperature is controlled at each time step. Decreasing the temperature in any node below the liquidus temperature $\left(\Theta_{L}\right)$ starts the procedure of shrinkage cavity formulation. The amount of the cavity depends on the value of solidification shrinkage coefficient $\left(S_{h}\right)$. On the base of known of the solid fraction volume $(\Phi)$ at the moment $(.)^{t}$ (previous step) and (. $)^{t+1}$ (current time step) the volumetric increment of the solid phase in the $i$-th node is calculated in the following way:

$$
\Delta V_{S(i)}=\left(\Phi_{S(i)}^{t+1}-\Phi_{S(i)}^{t}\right) V_{L(i)}^{t}
$$

Actual nodal volume of the solid phase is calculated using the equation below:

$$
V_{S(i)}^{t+1}=\Delta V_{S(i)}+V_{S(i)}^{t}
$$

Total volumetric increment of the solid phase in the casting is obtained as follows:

$$
\Delta V_{S}=\sum_{i=1}^{n} \Delta V_{S(i)}
$$

The nodal volume of the liquid phase is actualized according to the formula below:

$$
V_{L(i)}^{t+1}=V_{L(i)}^{t}-\Delta V_{S(i)}
$$

The corrected content of the solid phase in each node is obtained from:

$$
\Phi_{S(i)}^{t+1}=\frac{V_{S(i)}^{t+1}}{V_{(i)}}
$$

Global increment of the shrinkage cavity volume at current time step is determined as follows:

$$
\Delta V_{\text {shc }}=\Delta V_{S} S_{h}
$$

Equations shown above are executed at each time step causing gradual incrementation of the shrinkage cavity volume in the casting.

\section{Example of numerical calculations}

The calculations were carried out for the casting-mould-ambient system with the longitudinal section shown in Figure 1. The given dimensions of the essential elements of that system were as follows: $d=0.260 \mathrm{~m}, d_{o}=0.200 \mathrm{~m}, d_{n}=0.130 \mathrm{~m}$, $d_{1}=0.020 \mathrm{~m}, h=0.250 \mathrm{~m}, h_{o}=0.070 \mathrm{~m}, h_{n}=0.150 \mathrm{~m}$. 
The thermophysical properties of a cast steel alloy poured into a cast iron mould were taken from works $[6,9]$. The linear change of density $(\rho)$ and thermal conductivity $(\lambda)$ was assumed in $\Theta_{L^{-}} \Theta_{S}$ temperature interval. The variability of the dynamical viscosity coefficient $(\mu)$ with respect to temperature was determined according to the exponential relationship in range $0.003 \div 10^{5} \mathrm{Ns} / \mathrm{m}^{2}$. It is assumed here that the value of solidification shrinkage coefficient $\left(S_{h}\right)$ for considered alloy was equal to $3.85 \%$. The overheated metal with temperature $\Theta_{\text {in }}=1850 \mathrm{~K}$ and with velocity $v_{i n}=0.1 \mathrm{~m} / \mathrm{s}$ was poured into the mould with the initial temperature $\Theta_{M}=350 \mathrm{~K}$. The heat-transfer coefficient between mould and ambient $\alpha_{M}=100 \mathrm{~W} /\left(\mathrm{m}^{2} \mathrm{~K}\right)$ and thermal conductivity coefficients of the protective coating $\lambda_{G_{o}}=5$ and $\lambda_{G_{n}}=0.125 \mathrm{~W} /(\mathrm{mK})$ were assumed. The remaining characteristic temperatures were equal to: $\Theta_{L}=1810 \mathrm{~K}$, $\Theta_{S}=1760 \mathrm{~K}, \Theta_{a}=300 \mathrm{~K}, \Theta_{A}=350 \mathrm{~K}$. The flat free surface of metal was established and moved with net scale in consecutive time steps, which ensured that the continuity condition is satisfied.

a)

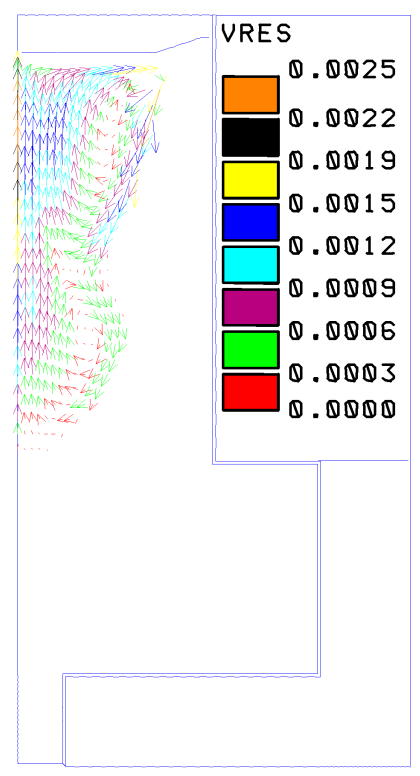

b)

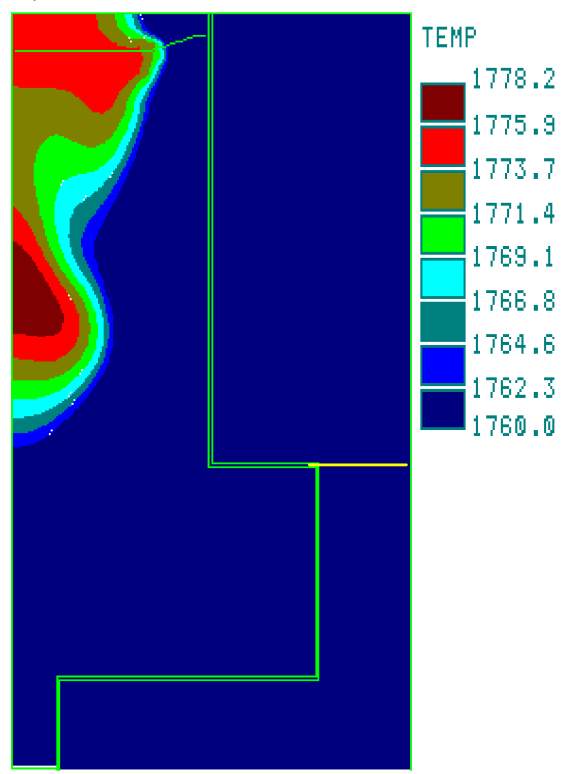

Fig. 2. Velocity field (a) and temperature field (b) after time $t=175 \mathrm{~s}$

Heat transfer and fluid flow phenomena, proceeding in the mould cavity during filling and after the filling is finished until the completion of solidification, were analysed. The influences of liquid metal movement inside the mould on the solidification kinetics of the casting were determined. In the numerical calculations, after the completion of filling of the mold cavity, the formation of the shrinkage cavity was estimated. In other steps of time the volume of the solid phase was determined, which allowed one to calculate the increase in volume shrinkage cavity, which was 
a symptom of the lowering of the liquid metal in the riser. In this way, the shape and depth of the shrinkage cavity were determined. Examples of calculation results are shown in the form of the temperature and velocity fields (Figs. 2, 3). So taking into account the mould filling and convectional motions of the molten metal, allows casting conditions are able to be evaluated in detail and position of the shrinkage cavity to be identified more precisely.

a)

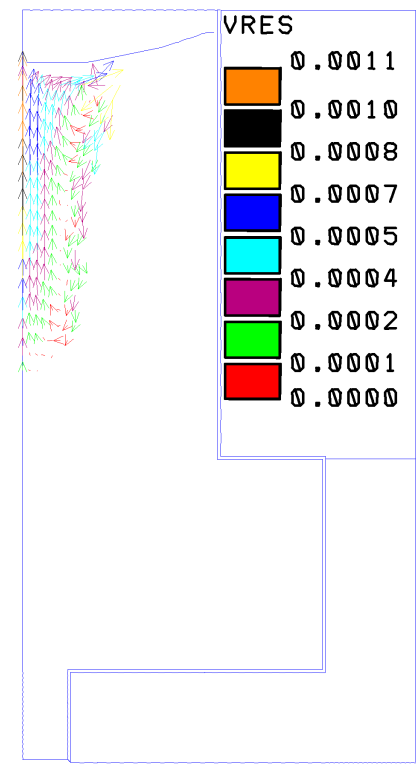

b)

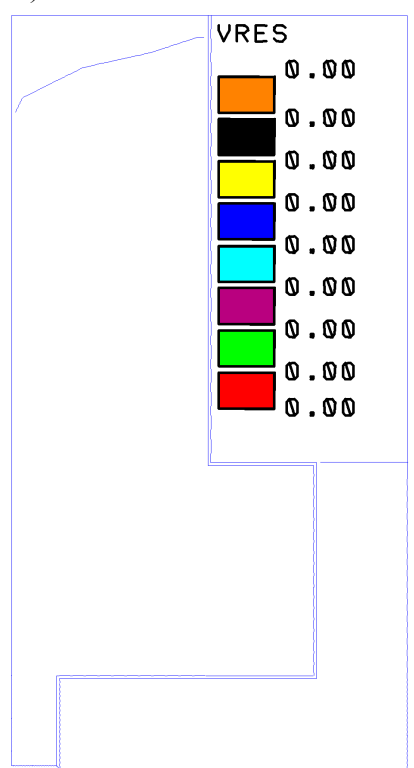

Fig. 3. Velocity field and shrinkage cavity area: a) $t=215 \mathrm{~s}$, b) $t=265 \mathrm{~s}$

\section{Conclusions}

This paper has presented the coupled model of solidification for the transient evaluation of fluid flow and heat transfer during the casting process. Numerical analysis included the filling process of mould cavity with molten metal, fluid flow, convection motions of alloy liquid phase, solidification process and shrinkage cavity formulation. After analysing the results obtained from the conducted numerical simulations, a non-uniform increment of solid phase in particular parts of the casting was noted. This increment depends on the intensity of liquid metal movement occurring in the casting. The end of solidification took place in the riser, which suggests that the feeder performed its function and that the remaining parts of the riser-casting system are free from shrinkage defects. The creation of the shrinkage cavity was observed in the top part of the riser. If the riser is cooled slowly, the final of the shrinkage cavity looks like a shallow cone, but if the cooling rate is high, the defect is much deeper $[2,4]$. The presented mathematical model and its numeri- 
cal implementation are able to predict the shape of shrinkage cavities which appear during the casting process. The choice of cooling parameters in conjunction with analysis of the results obtained from computer simulation may be helpful in selection of optimal parameters of the casting process. Appropriate control of the casting process parameters will result in shallow location of the potential defect, which is desired from the technological point of view.

\section{References}

[1] Dyja R., Sczygiol N., Domański Z., The formation of cavities in castings and their impact on the conditions of heat dissipation, Proceedings of the International Multi Conference of Engineers and Computer Scientists 2013, 2, 819-823.

[2] Skrzypczak T., Węgrzyn-Skrzypczak E., Simulation of shrinkage cavity formation during solidification of binary alloy, Archives of Foundry Engineering 2010, 10, 1, 147-152.

[3] Bockus S., Venckunas A., Zaldarys G., Simulation of a shrinkage cavity in the risers of ductile iron castings, Materials Science 2005, 11, 1, 19-23.

[4] Sowa L., Numerical simulation of the shrinkage cavity within solidifying steel casting, Archives of Foundry 2002, 2, 4, 245-250.

[5] Sowa L., Sczygiol N., Computer simulation of the filling and solidification of a gravity die casting, Solidification of Metals and Alloys 2000, 44, 2, 329-334.

[6] Węgrzyn-Skrzypczak E., Skrzypczak T., Mathematical and numerical basis of binary alloy solidification models with substitute thermal capacity. Part II, Journal of Applied Mathematics and Computational Mechanics 2014, 13, 2, 141-147.

[7] Bokota A., Domański T., Sowa L., Model and numerical analysis of mechanical phenomena of tools steel hardening, Archives of Foundry Engineering 2010, 10, 1, 19-22.

[8] Kukla S., Siedlecka U., Heat conduction problem in a two-layered hollow cylinder by using the Green's function method, Journal of Applied Mathematics and Computational Mechanics 2013, $12,2,45-50$.

[9] Mochnacki B., Suchy S.J., Numerical Methods in Computations of Foundry Processes, Polish Foundrymen's Technical Association, Kraków 1995. 